\title{
Histopathological Characteristics of Atrophic Gastritis in Adult Population
}

\author{
Marija Milićević, Snežana Božanić and Nenad Šolajić \\ Department of Pathology, Faculty of Medicine, University of Novi Sad, Novi Sad 21000, Serbia
}

\begin{abstract}
Introduction: Chronic gastritis is inflammation of the gastric mucosa. It can be non-atrophic and atrophic. Atrophy is defined as the loss of appropriate glands. It is frequently located in antral mucosa as consequence of Helicobacter pylori infection and it is associated with intestinal gastric cancer. Goal: Describe histopathological and demographic characteristics of atrophic gastritis. Matherial and methods: We assessed the pathological reports of 100 patients with atrophic gastritis whose characteristics were evaluated by using a semiquantitative scale of Sidney system of classification of gastritis. To assess the significance between the incidence of various parametres we used $\chi^{2}$ test. Results: We found that the difference in frequency of atrophic gastritis between men and women was not statistically significant. The difference in distribution is statistically significant in favor of the antrum. Among patients who have atrophy with Helicobacter pylori infection and intestinal metaplasia and those who do not have metaplasia, it was found that the difference is highly statistically significant. Conclusion: The most frequent localisation of atrophic gastritis is antral mucosa. There is no difference between men and women in frequency of atrophic gastritis, while the aging is related with more often occurrence of atrophic gastritis.
\end{abstract}

Key words: Gastritis, atrophic, metaplasia, Helicobacter pylori.

\section{Introduction}

Gastritis is an inflammation of the gastric mucosa. It can be acute or chronic. According to the pathological characteristics, chronic gastritis is divided into atrophic and non-atrophic gastritis [1,2]. Atrophy of the gastric mucous represents a complete or partial loss of normal gastric glands (Fig. 1). Glands which are lost can be replaced by connective tissue, or with foveola that are coated with intestinal metaplastic epithelium and/or with metaplastic mucous glands (pseudo pyloric metaplasia, pyloric metaplasia, mucous metaplasia) [3, 4]. Atrophy can be categorized as mild, moderate and severe based on rough estimates of the thickness of the glandular mucous layer to the total thickness of the mucosa.

Atrophic gastritis is often localized in the antral mucosa and it is associated with Helicobacter pylori

Corresponding author: Marija Milićević, postgraduate, reserch field: pathology and infectious diseases. E-mail: novisad56@gmail.com.
(H. pylori) infection. Isolated occurrence of atrophy in the mucosa of the corpus is mainly associated with autoimmune processes. Multifocal atrophic gastritis is mainly present in populations living in sub-optimal sanitary conditions, including mainly residents of southern and eastern Asia, Latin America and all parts of central, southern and eastern Europe. Atrophic pangastritis represents the advanced stage of multifocal atrophic gastritis and a high risk of developing cancer [5, 6].

Chronic gastritis may be caused by $H$. pylori infection, immunological factors, radiation or thermal injury, and prolonged use of alcohol, aspirin and other irritating drugs $[7,8]$.

Chronic atrophic gastritis plays an important role in the development of intestinal type of gastric cancer which is the most common type of gastric cancer and is one of the leading causes of death worldwide [9].

The World Congress of Gastroenterology in Sydney in 1990 established the guidelines for the 
classification of gastritis, which use a semi-quantitative estimation and tables to show the type

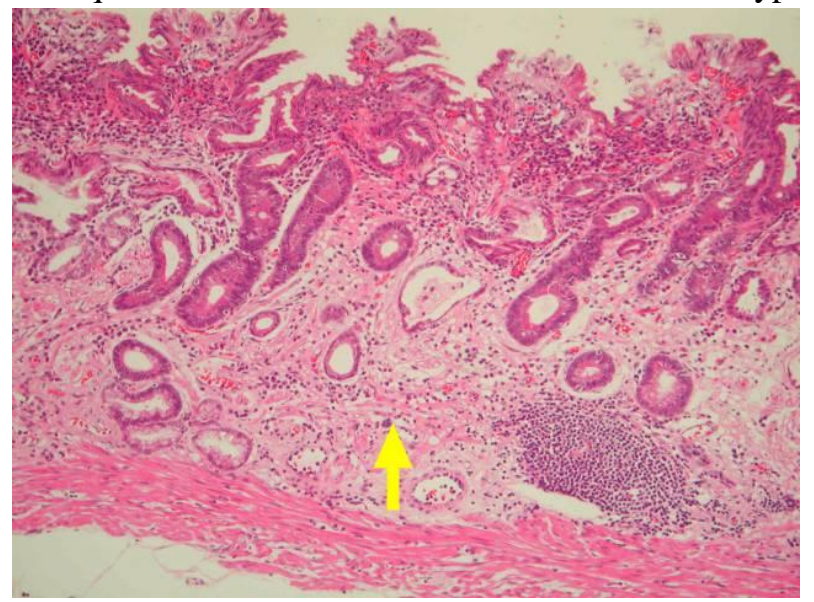

Fig. 1 Atrophic gastritis, 10x enlarged, HE coloring [11].

of mucosal inflammation, atrophy, activity, intestinal metaplasia and HLO (Helicobacter-like organisms) on a scale of 0 to 3. Significant pathological changes that are not covered by the table (foveolar hyperplasia, dysplasia, the presence of lymph follicles, granuloma, etc.) are note down separately. This classification has been revised four years later (Houston Gastritis Workshop, 1994) [10].

The aims of the work are: (1) Show pathological characteristics of atrophic gastritis; (2) Show demographic characteristics (gender, age) of atrophic gastritis; (3) Examine the importance of the region (antrum, corpus) in atrophic gastritis; (4) Examine the significance of intestinal metaplasia in atrophic gastritis; (5) Examine the significance of intestinal metaplasia in atrophic gastritis associated with $H$. pylori infection.

\section{Materials and Methods}

In this retrospective study we used data from the registers from Clinical Center of Vojvodina, the Center for pathology and histology. Data that we used were already analyzed stomach biopsies by a pathologist. We assessed the pathological reports of 100 patients with atrophic gastritis whose characteristics pathologist evaluated using semiquantitative scale of Sydney classification system of gastritis. According to the classification, the following parameters are analyzed: type of mucosa, inflammation, atrophy, activity of inflammation, intestinal metaplasia and the presence of $H$. pylori in the mucosa on a scale of 0 to 3 . Assessment of the above parameters is performed on endoscopic biopsy material obtained (two biopsies from the antrum and from the corpus). Biopsy specimens were fixed in formalin, dehydrated, embedded in paraffin according to standard histological procedures and stained with HE (hematoxylin methods-eosin), Giemsa, PAS (periodic acid-Schiff) and Alcian blue.

The collected data were statistically analyzed, tabulated and graphically presented using the software package Microsoft Office. For statistical analysis are used the mean ( \pm ) standard deviation (SD). To assess the significance of frequency between various parameters used the $\chi^{2}$ test.

\section{Results}

Study included 100 patients, of whom 53 were men and 47 women as it is shown in Figs. 2 and 3.

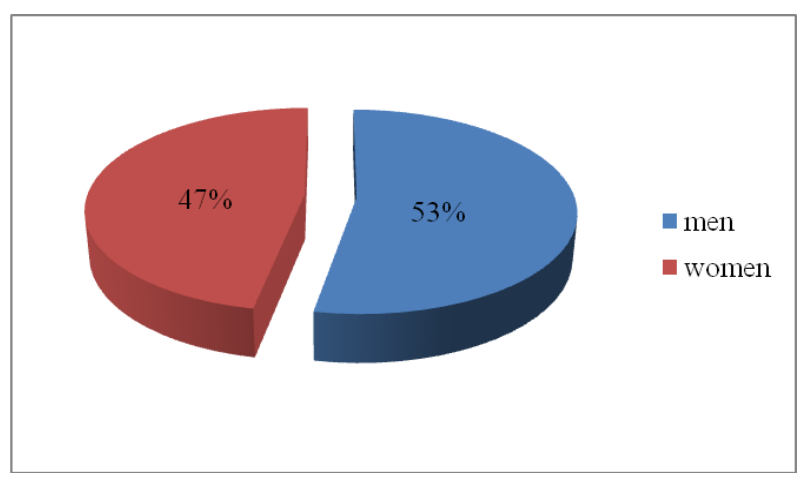

Fig. 2 Distribution of patients according to the gender. 


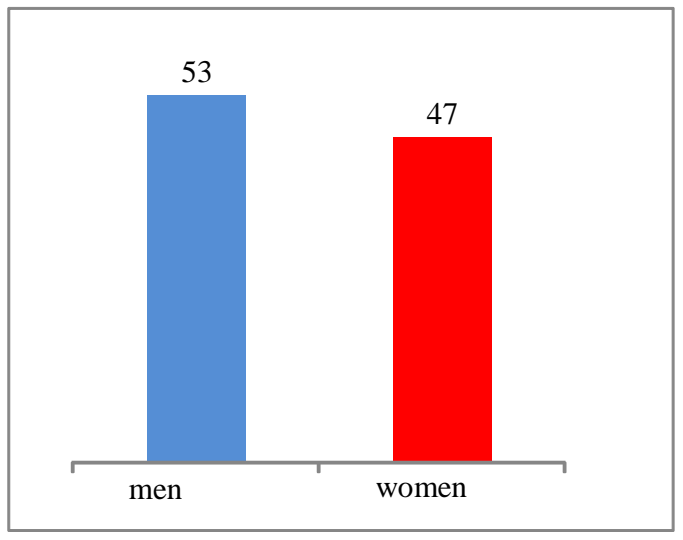

Fig. 3 Distribution of atrophic gastritis by gender.

With using the $\chi^{2}$ test, the difference in the incidence of atrophic gastritis in men and women was tested and it was found that the difference in frequency was not statistically significant $(\chi 2=0.25$ which is less than the tabular value of $p=0.05)$.

The average age of the patients was $63.79 \pm 13.5$ years. The average age of the men was $63.83 \pm 12.56$ years and for women was $63.74 \pm 14.63$ years. The oldest patient had 87 years, and the youngest was 30 years old.

The total number of histopathological preparations was 202 , because two patients $(2 \%)$ had 3 instead of 2 biopsies (two were from the antrum and one from the corpus). Of this number, 106 belonged to men $(52.47 \%)$ and 96 women (47.53\%). In 97 patients biopsies were taken from each region of the stomach, while two patients had only biopsy from antrum, and only 1 patient had biopsy only from corpus.

Of the 100 analyzed patients with atrophic gastritis, in 66 patients $(66 \%)$ atrophy was observed only in the area of the antrum, in 11 patients $(11 \%)$ only in the area of the corpus, and in 23 patients (23\%) was observed in both regions.
In 75 histopathological preparations atrophy has not been found, while in the examination of 127 histopathological preparations atrophy has been found (93 were located in the antrum, and 34 in the corpus as it is shown in Fig. 4).

Representation of different localization was tested using the $\chi 2$ test and it has been found that the difference is highly statistically significant $(\chi 2=26.48$ which is larger than the tabular value of $p=0.01$ ).

Atrophy was diagnosed in the 127 biopsies, intestinal metaplasia was found in 45 samples, while 82 samples have been without the presence of metaplasia as it is shown in Fig. 5.

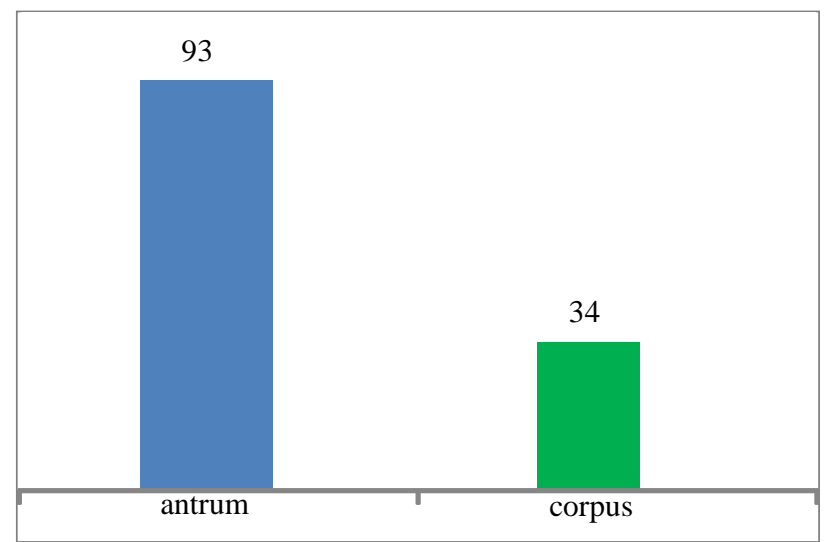

Fig. 4 The presence of atrophic gastritis in relation with the localization. 


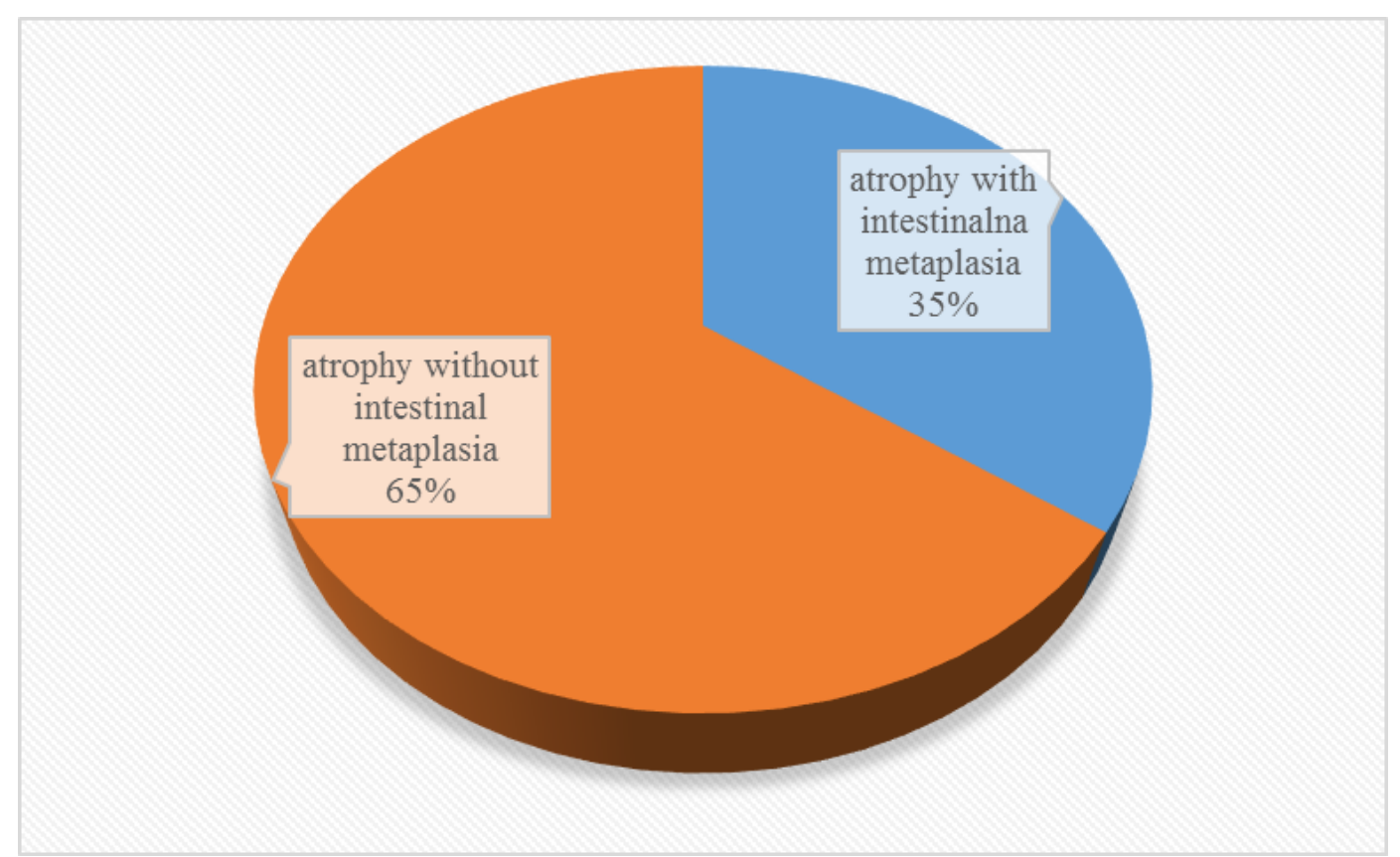

Fig. 5 Relation between atrophic gastritis with and without intestinal metaplasia.

The significance of differences between groups of patients with intestinal metaplasia and without intestinal metaplasia was tested and it was found that the difference is statistically significant $\left(\chi^{2}=10.2\right.$ which is greater than the tabular value of $p=0.01$ ).

The significance of differences between patients who have atrophy with $H$. pylori infection and intestinal metaplasia and those who do not have metaplasia was tested and it was found that the difference is highly statistically significant $\left(\chi^{2}=14.02\right.$ which is larger than the tabular value of $p=0.01$ and it is shown in Fig. 6).

\section{Discussion}

During the study we analyzed 100 biopsy specimens of atrophic gastritis whose characteristics were evaluated by a pathologist with the use of the scale recommended by the Sydney classification system.

Sydney classification system of gastritis involves two main divisions, histological and endoscopic. Histological analysis of biopsies of the stomach has three parts: the topography (corpus, antrum or full stomach), morphology (inflammation, atrophy, activity of inflammation, intestinal metaplasia and density of $H$. pylori in the mucosa on a scale of 0-3) and etiology. The scale that is used is visual - analog and recommended by the Sydney classification system. To make this classification applicable, it must be taken at least two biopsies from the corpus (front and rear wall) and from the antrum ( $2 \mathrm{~cm}$ proximal to the pylorus). Each focal change should be biopted. Biopsies from the corpus and antrum are submitted to histopathological examination in separate containers and well marked. [5, 8].

The amended Sydney system divides gastritis into non atrophic gastritis (caused by Helicobacter and other causes), atrophic (autoimmune, caused by Helicobacter, caused by environmental factors), and special forms of gastritis (chemical, radiation, lymphocyte, noninfectious, granulomatous, eosinophilic and other infectious pathogens except $H$. pylori). The presence of mononuclear inflammatory infiltrate (lymphocytes and plasma cells) in the lamina propria is the only condition for the diagnosis of chronic gastritis [8].

Atrophy of the gastric mucosa can occur at any age of adult population, but the prevalence increases with 
age of patients [12]. Data obtained during the study, the literature and observed that the average age of presented in table 1 , corresponding to the data from patients was $63.79 \pm 13.5$ years.

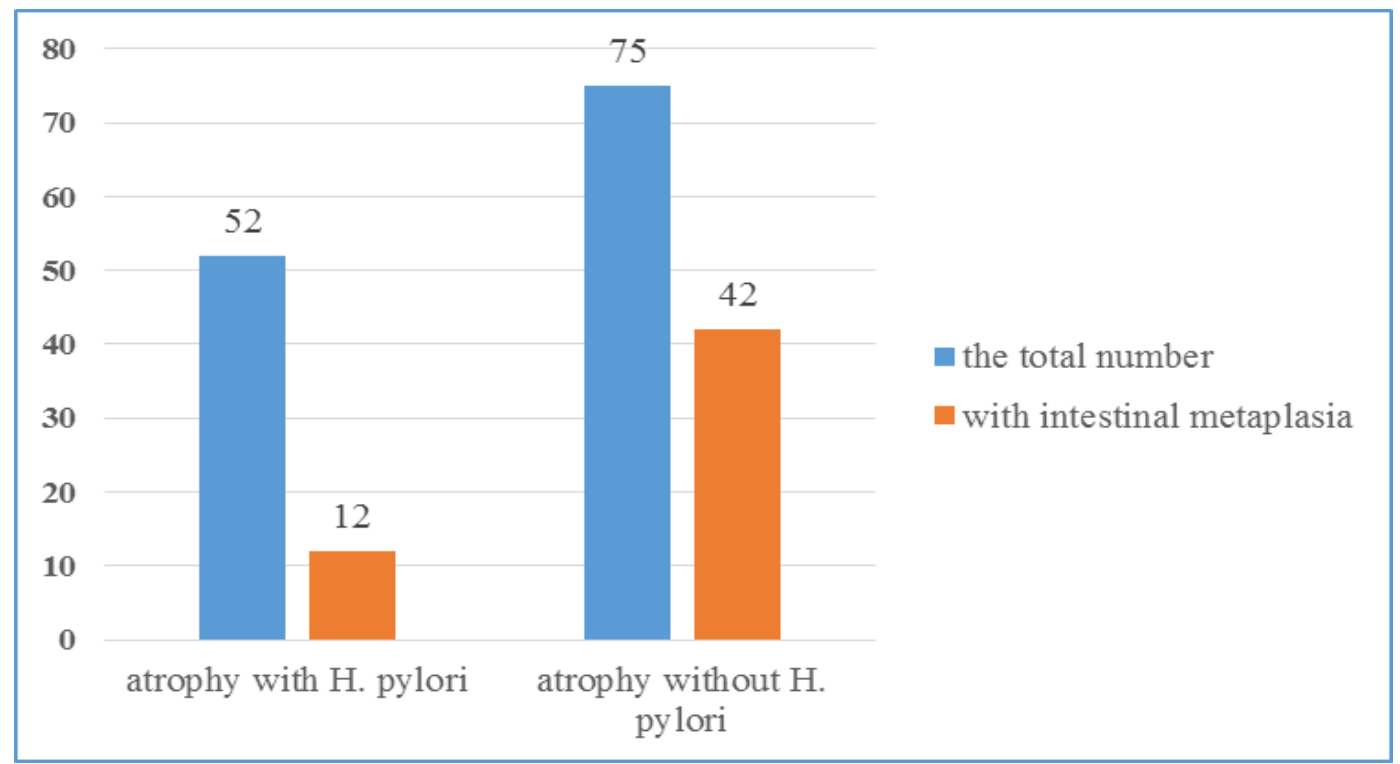

Fig. 6 Relation between $H$. Pylori infection and intestinal metaplasia in atrophic gastritis.

Table 1 Distribution of patients with atrophic gastritis by age.

\begin{tabular}{lcccc}
\hline $\begin{array}{l}\text { Age } \\
\text { (years) }\end{array}$ & number of patients & Relative frequency $(\%)$ & Cumulative frequency $(\%)$ & Average age (years) \\
\hline $28-35$ & 3 & 3 & 3 & 32 \\
$36-43$ & 7 & 7 & 10 & 39 \\
$44-51$ & 8 & 8 & 18 & 48.12 \\
$52-59$ & 17 & 17 & 35 & 56.41 \\
$60-67$ & 24 & 24 & 59 & 63.71 \\
$68-75$ & 17 & 17 & 76 & 72 \\
$76-83$ & 22 & 22 & 98 & 79.09 \\
$84-91$ & 2 & 2 & 100 & 86.5 \\
Total & 100 & 100 & & \\
\hline
\end{tabular}

The incidence of atrophic gastritis in relation to gender did not show statistically significant difference between men and women, as it is shown in Fig. 2.

Analysis of the data on the localization of atrophic gastritis showed statistically significant difference compared to the antrum and corpus of the stomach and it is displayed in Fig. 3. Of the 127 biopsies with atrophic changes, 93 was diagnosed in the antrum, and 34 in the gastric corpus. The data that are shown correspond to the literature in which it is mentioned that in areas which do not fall within the areas of suboptimal social conditions of life, the most common cause of atrophic gastritis, H. pylori infection and that changes are localized in the gastric antrum [5].
Intestinal metaplasia is the most commonly found in $H$. pylori-positive people. The absence of $H$. pylori in the stomach with extensive intestinal metaplasia can be explained by changing the biochemical milieu where the lining of $H$. pylori can not be adjusted. Although the presence of $H$. pylori in these patients has not been detected, probably infection with $H$. pylori existed before the changes that were caused by the infection. Our finding of a statistically significantly smaller number of patients that have both intestinal metaplasia and $H$. pylori detected, than those who have intestinal metaplasia without H.pylori infection, is consistent with findings in the literature $[8,12]$. Intestinal metaplasia may occur in 
autoimmune atrophic gastritis, when changes are seen almost exclusively in the corpus, or only rarely in the antrum in some special form of gastritis. The clinical significance of intestinal metaplasia refers to its ability to progress to dysplasia and adenocarcinoma.

It is thought that $H$. pylori infection is a major cause of chronic antral gastritis (a condition that is present in more than $35 \%$ of asymptomatic adult population), and less attention is given to other risk factors: alcohol, coffee, smoking, chronic exposure to certain substances (potassium, iron, non-steroidal anti-inflammatory drugs), physical-chemical agents in foods, foods high in nitrates and salt, food which is scarce in green vegetables and fresh fruits, coal dust and other environmental factors [8, 12].

A special clinical significance of chronic $H$. pylori gastritis is what carries an increased risk for duodenal and gastric ulcers, gastric cancer and lymphoma $[4,8$, 9].

\section{Conclusions}

Gender of patients does not have the influence on the incidence of atrophic gastritis.

- frequency of atrophic gastritis increases with age of patients.

- Atrophic gastritis is more often localized in the antral mucosa than in the corpus.

- Atrophic gastritis is often accompanied by $H$. pylori infection.

- The presence of intestinal metaplasia reduces the probability of finding $H$. pylori

\section{Literature}

[1] Rugge, M., Meggio, A., Pennelli, G., Piscioli, F., Giacomelli, L., Pretis, G., and Graham, D. Y. 2007. "Gastritis Staging in Clinical Practice: the OLGA Staging System.“ Gut 56: 631-6.

[2] Rugge, M., Pennelli, G., Pilozzi, E., Fassan, M.,
Ingravallo, G., Russo, V. M., and Di Mario, F. 2011. "Gastritis: The Histology Report." Digestive and Liver Disease 43(4):373-84.

[3] Ricuarte, O., Gutierrez, O., Cardona, H., Kim, J G., Graham, D. Y., and El-Zimaity, H. M. 2005. "Atrophic Gastritis in Young Children and Adolescents." Journal of Clinical Pathology 58: 1189-93.

[4] Rugge, M., Kim ,J G., Mahachai, V., Miehlke, S., Pennelli ,G., Russo, V M., Pernq, C. L., Chang, F. Y., Tandon, R. K., Singal, J. J., Sung, J. J., Valenzuela, J. E., Realdi, G., Dore, M. P., and Graham, D. Y. 2008. "OLGA Gastritis Staging in Young Adults and Countru-Specific Gastritic Cancer Risk." International Journal of Surgical Pathology 2: 150-4.

[5] Rugge, M., and Genta, R. M. 2005. " Staging and Grading of Chronic Gastritis." Human Pathology 36: 228-33.

[6] Fareed, R., Abbas, Z., and Shah, M. A. 2000. "Effect of Helicobacter Pylori Density on Inflammatory Activity in Stomach". The Journal of the Pakistan Medical Association 50: 148.

[7] Dimarino, A. 2010. Gastrointestinal Diseases. Beograd: A.B.D.Profendo, 279-362.

[8] Pejin, D. 2009. Internal Medicine II. Novi Sad: Faculty of Medicine, 1005-19.

[9] Weck, M. N., and Brenner, H 2006. "Prevalence of Chronic Atropic Gastritis in Different Parts of the World." Cancer Epidemiology, Biomarkers and Prevention 15 (6): 1083-94.

[10] Öztürk, S., Serinsöz, E., Kuzu, I., Ensari, A., Erden, E., and Kansu, A. 2001.'The Sydney System in the Assessment of Gastritis: Inter-observer Agreement." The Turkish Journal of Gastroenterology12: 36-9.

[11] Pathology Web [home page on the Internet]. Taipei, Tajvan: Pathology Departmnent of TMU; 2009 Available from: ttp//pathology.tmu.edu.tw/mocroskopy/showall.asp?=2033.

[12] Ohkuma, K., Okada, M., Murayama, H., Seo, M., Maeda, K., Kanda, M., and Okabe, N. 2000. "Association of Helicobacter pylori Infection with Atrophic Gastritis and Intestinal Metaplasia." Journal of Gastroenterology and Hepatology 15: 1105-12. 